Research Article

\title{
Discrimination and Geographical Origin Prediction of Cynomorium songaricum Rupr. from Different Growing Areas in China by an Electronic Tongue
}

\author{
Jiaji Ding $\mathbb{D}^{1,2}$ Caimei Gu, ${ }^{2}$ Linfang Huang $\mathbb{D}^{2},{ }^{2}$ and Rui Tan $\mathbb{D}^{1}$ \\ ${ }^{1}$ College of Medcine, Southwest Jiaotong University, Chengdu 610031, China \\ ${ }^{2}$ Institute of Medicinal Plant Development, Chinese Academy of Medical Sciences \& Peking Union Medical College, \\ Beijing 100193, China \\ Correspondence should be addressed to Linfang Huang; 15801545922@139.com and Rui Tan; tanrui@home.swjtu.edu.cn
}

Received 11 September 2018; Accepted 31 October 2018; Published 22 November 2018

Academic Editor: Jaroon Jakmunee

Copyright (c) 2018 Jiaji Ding et al. This is an open access article distributed under the Creative Commons Attribution License, which permits unrestricted use, distribution, and reproduction in any medium, provided the original work is properly cited.

Cynomorium songaricum Rupr. is a well-known and widespread plant in China. It has very high medicinal values in many aspects. The study aimed at discriminating and predicting C. songaricum from major growing areas in China. An electronic tongue was used to analyze C. songaricum based on flavor. Discrimination was achieved by principal component analysis and linear discriminant analysis. Moreover, a prediction model was established, and C. songaricum was classified by geographical origins with $100 \%$ degree of accuracy. Therefore, the identification method presented will be helpful for further study of C. songaricum.

\section{Introduction}

Cynomorium songaricum Rupr. of the family Cynomoriaceae is a desert, holoparasitic perennial plant found in China, Mongolia, Iran, and Afghanistan [1, 2]. In China, C. songaricum grows in Xinjiang, Inner Mongolia, Ningxia, Qinghai, and Gansu [3] (Figure 1). C. songaricum, called Suo Yang in China, is a known food, nutrient, and a tonic herb for improving kidney and immunity function and treating constipation [4,5]. This plant is one of the most popular herbs in the world and is documented in some famous medicinal works [1]. Various compounds, including flavonoids, organic acids, steroids, saccharides, terpenoids, phloroglucinol adducts, phenylpropanoids, and other types of compounds, have been isolated from C. songaricum to date $[1,6]$. These chemical compounds exhibit numerous biological activities, including antiapoptosis, antifatigue, antioxidant, antiosteoporotic, antiaging, antidiabetic, antiHIV protease, anti-HCV protease, and fertility promotion [1, 7-9].

In our previous work, we presented that the chemical constituents of $C$. songaricum from different producing areas vary, thus affecting the quality of the plant [2]. Genuine medicinal herb, which means Daodi yaocai in Chinese, is a unique definition in traditional Chinese medicine. Medicinal herbs growing in a specific place exhibit high quality [10]. Currently, chromatographic herbal fingerprints have become one of the most applied quality control tools for similarity analyses of herbal medicines [11]. However, it costs a relatively long time. Thus, a more convenient way for identification and quality control of herbs is needed.

Electronic tongues are analytical systems formed from an array of electrochemical sensors combined with dataprocessing tools intended to interpret electrochemical signals. Similar to human receptors, the sensors of an electronic tongue undergo a series of reactions. While the generated reactions differ from one another, the information acquired from each sensor is complementary. Then, the results combined by the sensors generate a unique fingerprint that can reflect the macroscopic characteristics of samples. In biological mechanisms, gustatory signals are transducted by brain nerves in the form of electric signals. Electronic tongue sensors approach flavors similarly, given that electric signals 


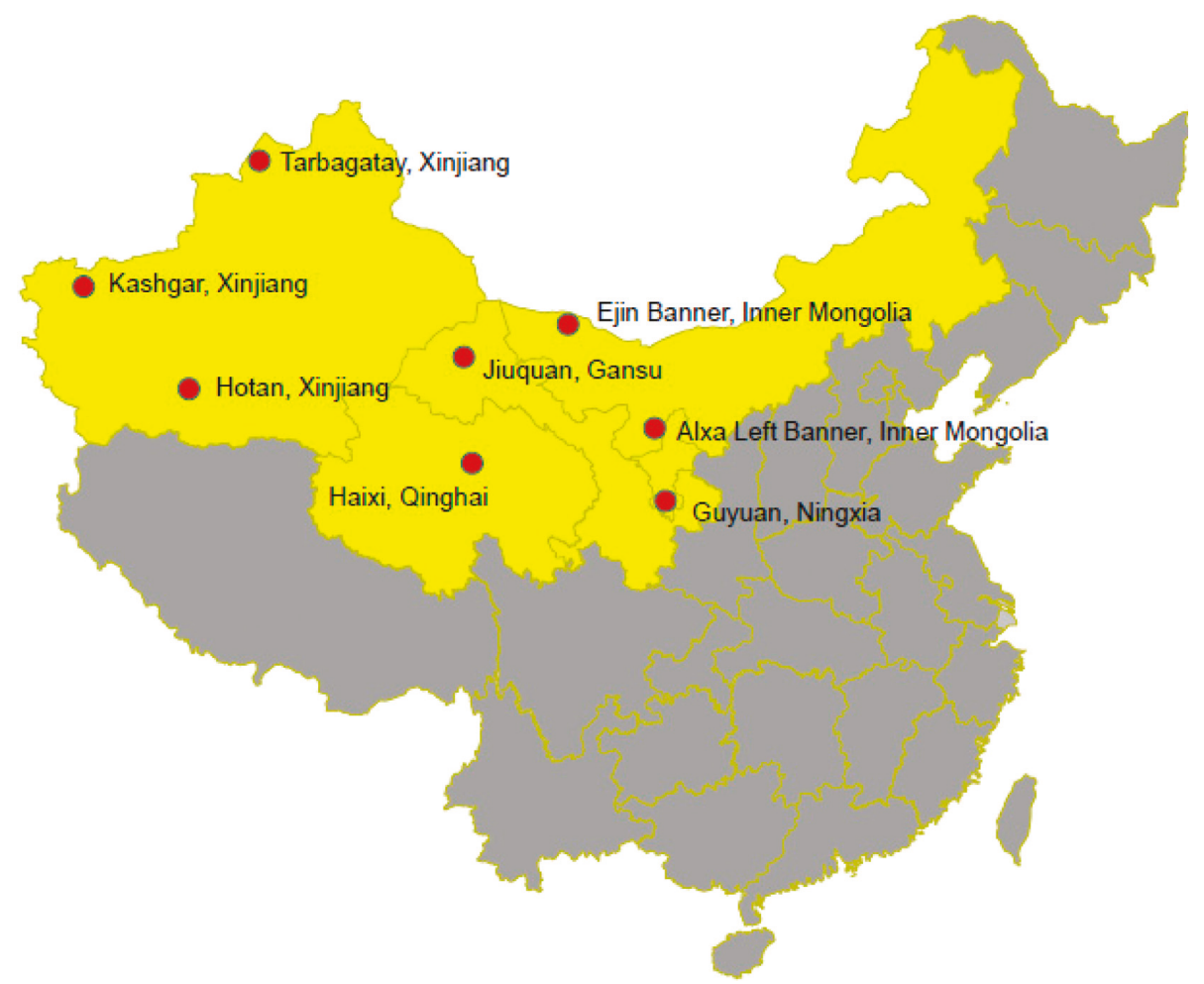

Figure 1: The distribution of Cynomorium songaricum Rupr. in China.

are generated with potentiometric variations. The perception and recognition of taste quality are based on the recognition or building of activated sensory nerve patterns in the brain and the gustation fingerprint of a product. This step is accomplished by the statistical software of the electronic tongue that can translate sensor data into taste patterns [12-19]. In the recent years, electronic tongues have been commonly used to analyze food and beverages, given their advantages of short response time, strong objectiveness, human safety, and repeatability [20]. As for some herbs like C. songaricum, they have different tastes and flavors according to different places of origin and are ready to eat. Based on this, the simplicity and convenience of electronic tongues could be used in the analysis of the herbs.

In this work, we first developed a method to discriminate and predict the geographical origin of C. songaricum from different growing areas in China by using an electronic tongue. Pattern recognition techniques, including principal component analysis (PCA) and linear discriminant analysis (LDA), were used for data analysis in this research. In addition, this study provided a simple approach for identifying the geographical origins of $C$. songaricum, and the acquired information can be used for evaluating the quality of $C$. songaricum growing in China.

\section{Materials and Methods}

2.1. Samples. C. songaricum samples were collected from different areas in China (Kashgar in Xinjiang, Tarbagatay in Xinjiang, Jiuquan in Gansu, Guyuan in Ningxia, Hotan in Xinjiang, Haixi in Qinghai, Ejin Banner in Inner Mongolia, and Alxa Left Banner in Inner Mongolia) (Table 1). All of the samples were authenticated by Professor Linfang Huang in the Institute of Medicinal Plant Development, Chinese Academy of Medical Sciences, and Peking Union Medical College, Beijing, China.

2.2. Instrument. The electronic tongue system (taste sensing system Astree II, France) consists of a reference electrode and seven liquid sensors (ZZ, JE, BB, CA, GA, HA, and JB) with a cross-selection function, a fully automated sample injector, and a personal computer with a software for sample injection, data acquisition, and chemometric analysis.

2.3. Experimental Procedures. Pieces of each sample (10 g) were placed in a beaker, soaked with $200 \mathrm{~mL}$ of pure water for $30 \mathrm{~min}$, and then decocted for $30 \mathrm{~min}$. The solution was filtered immediately. The residue was processed according to the abovementioned method twice. Afterward, all filtrates were combined. The obtained solution was placed into the special beaker of the electronic tongue and detected at room temperature.

Each sensor collected data from each sample for $120 \mathrm{~s}$ and was cleaned for $10 \mathrm{~s}$. Then, data were recorded by the data acquisition system. All assays were carried out in triplicate.

2.4. Pattern Recognition. In this paper, PCA and LDA were used to differentiate $C$. songaricum originating from different places. 
TABLE 1: Sample list of Cynomorium songaricum Rupr.

\begin{tabular}{|c|c|}
\hline Sample & Place of origin \\
\hline \multicolumn{2}{|l|}{ Discrimination } \\
\hline $\mathrm{KX}-1$ & Kashgar, Xinjiang \\
\hline KX-2 & Kashgar, Xinjiang \\
\hline TX-1 & Tarbagatay, Xinjiang \\
\hline TX-2 & Tarbagatay, Xinjiang \\
\hline TX-3 & Tarbagatay, Xinjiang \\
\hline JG-1 & Jiuquan, Gansu \\
\hline JG-2 & Jiuquan, Gansu \\
\hline GN-1 & Guyuan, Ningxia \\
\hline GN-2 & Guyuan, Ningxia \\
\hline GN-3 & Guyuan, Ningxia \\
\hline HX-1 & Hotan, Xinjiang \\
\hline $\mathrm{HX}-2$ & Hotan, Xinjiang \\
\hline $\mathrm{HX}-3$ & Hotan, Xinjiang \\
\hline HQ-1 & Haixi, Qinghai \\
\hline HQ-2 & Haixi, Qinghai \\
\hline HQ-3 & Haixi, Qinghai \\
\hline EBIM-1 & Ejin Banner, Inner Mongolia \\
\hline EBIM-2 & Ejin Banner, Inner Mongolia \\
\hline EBIM-3 & Ejin Banner, Inner Mongolia \\
\hline ALBIM-1 & Alxa Left Banner, Inner Mongolia \\
\hline ALBIM-2 & Alxa Left Banner, Inner Mongolia \\
\hline \multicolumn{2}{|l|}{ Prediction } \\
\hline $\mathrm{KX}-3$ & Kashgar, Xinjiang \\
\hline $\mathrm{KX}-4$ & Kashgar, Xinjiang \\
\hline TX-4 & Tarbagatay, Xinjiang \\
\hline TX-5 & Tarbagatay, Xinjiang \\
\hline JG-3 & Jiuquan, Gansu \\
\hline JG-4 & Jiuquan, Gansu \\
\hline GN-4 & Guyuan, Ningxia \\
\hline GN-5 & Guyuan, Ningxia \\
\hline $\mathrm{HX}-4$ & Hotan, Xinjiang \\
\hline HX-5 & Hotan, Xinjiang \\
\hline HQ-4 & Haixi, Qinghai \\
\hline HQ-5 & Haixi, Qinghai \\
\hline EBIM-4 & Ejin Banner, Inner Mongolia \\
\hline EBIM-5 & Ejin Banner, Inner Mongolia \\
\hline ALBIM-3 & Alxa Left Banner, Inner Mongolia \\
\hline ALBIM-4 & Alxa Left Banner, Inner Mongolia \\
\hline
\end{tabular}

PCA is a multivariate statistical method that reduces the dimensionality of data while retaining most of the variation in the data [21]. This approach was created before World War II but became widely used during the "Quantitative Revolution" in the 1960s [22]. New linear combinations of variables are created to accomplish the reduction. The combinations, called principal components, characterize the objects studied and satisfy certain statistical and mathematical conditions. Thus, samples can be displayed by few variables, and assessment of similarities and differences among samples is simplified. Thus, PCA is a suitable method to discriminate different samples and is extensively applied in food and drug analysis.

LDA is another commonly used technique for data discrimination and dimensionality reduction. LDA is strongly linked to regression analysis and analysis of variance (ANOVA), which also aims at expressing one dependent variable as a combination of other measurements or features [23]. The method maximizes the ratio of the between-class distance to the within-class distance to guarantee maximum discrimination. LDA has been used in numerous applications, such as image retrieval, microarray data classification, face recognition, and food and beverage discrimination [24-27].

\section{Results and Discussion}

3.1. Radar Map. Figure 2 shows the radar map of samples from different places in China. The sensor response of the electronic tongue varied with the change in geographical origins. Evidently, sensors $\mathrm{BB}, \mathrm{CA}$, and $\mathrm{ZZ}$ show strong signals to the samples. In particular, sensor $B B$ exhibits the strongest response to the samples. Figure 2(a) shows the distinction among all samples from different places clearly. Signals from different samples show a considerable difference. Figure 2(b) illustrates the signals of every sample separately. Samples KX, TX, and HX are different from others, with the signals of sensor ZZ of these samples not exceeding 1000. Shapes of the radar maps of other samples are similar.

3.2. Principal Component Analysis. The first discrimination model was established using PCA to visualize the different $C$. songaricum groups where possible. The accumulated explained variance was $89.6 \%$, which was distributed in $79.5 \%$ (PC1) and $10.1 \%$ (PC2). Figure 3 shows the results of PCA score plot, and several trends are observed. Eight types of C. songaricum samples can be classified in general. Moreover, C. songaricum samples from Xinjiang are discriminated clearly between samples from other provinces. Similar samples appear in the same location of the graph. Thus, C. songaricum samples from Gansu, Ningxia, Qinghai, and Inner Mongolia are similar. The chemical constituents of these samples may be similar as well.

3.3. Linear Discriminant Analysis. Figure 4 shows the dispersion of C. songaricum samples by the LDA model. Compared with the PCA model, the LDA model shows a clearer discrimination among the eight types of C. songaricum. The explained variances by each discriminant function (DF) were 96.0\% (DF1) and 2.9\% (DF2). Each group of $C$. songaricum samples can be distinctly classified with others. As a result, the LDA model is a superior method to discriminate C. songaricum from different growing areas in China.

Given the good discrimination feature of the model, we used the LDA model to predict the geographical origin of unknown C. songaricum samples. As shown in Table 2, the prediction model can classify C. songaricum by geographical origins with $100 \%$ degree of accuracy.

\section{Conclusions}

We applied an electronic tongue to classify and predict $C$. songaricum samples from different places of origin. PCA and LDA were used for discrimination. The LDA model shows a clearer discrimination than the PCA model. The eight 


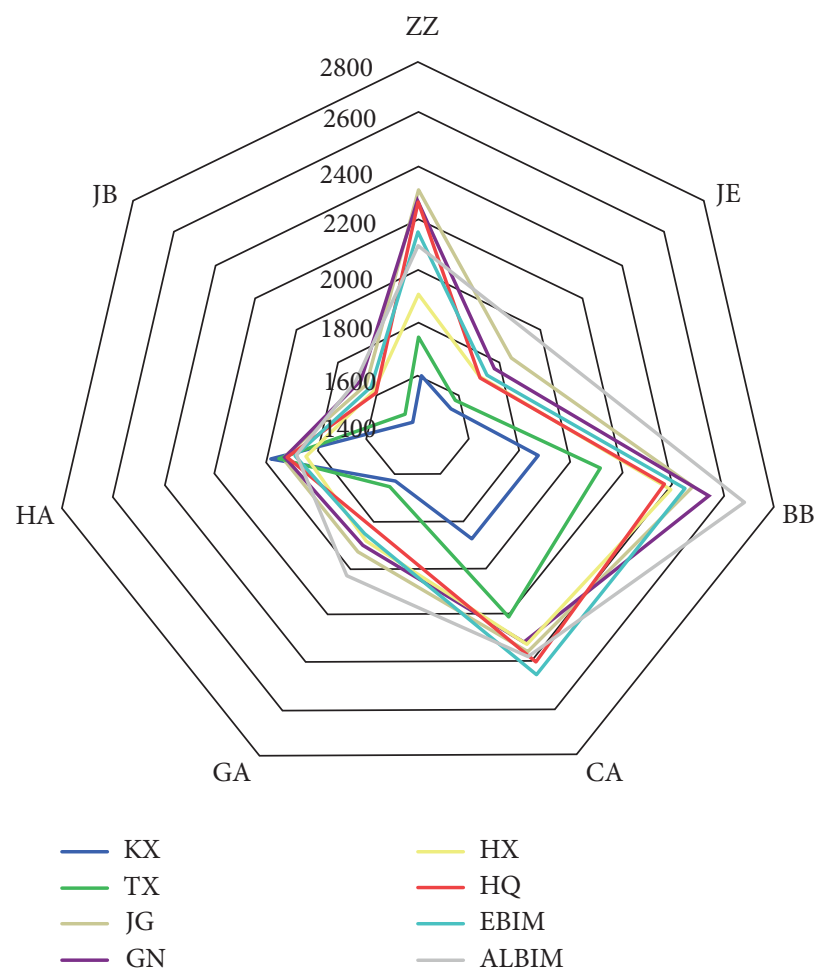

(a)
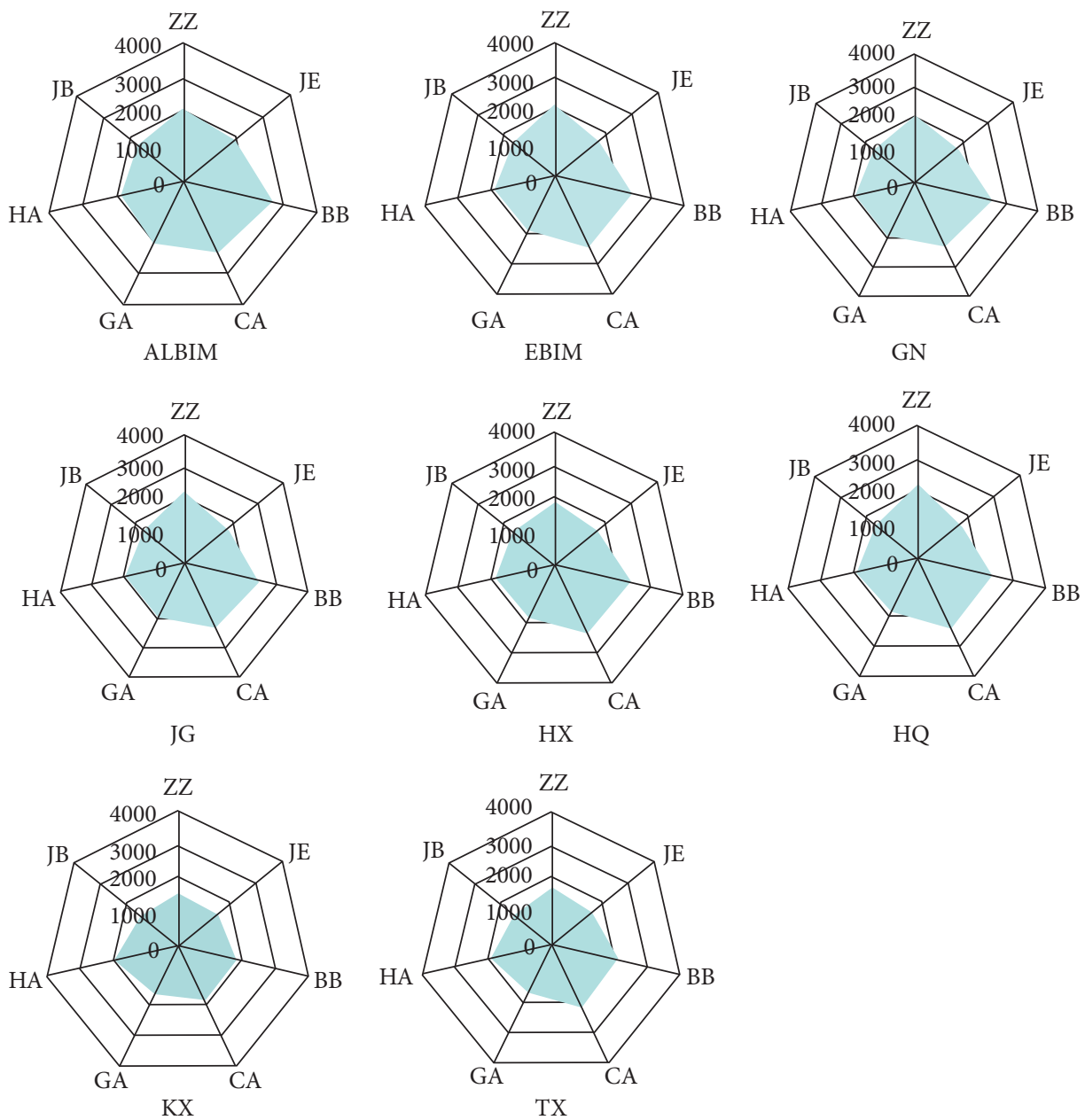

(b)

FIgURE 2: The radar map of C. songaricum from different producing areas in China. 


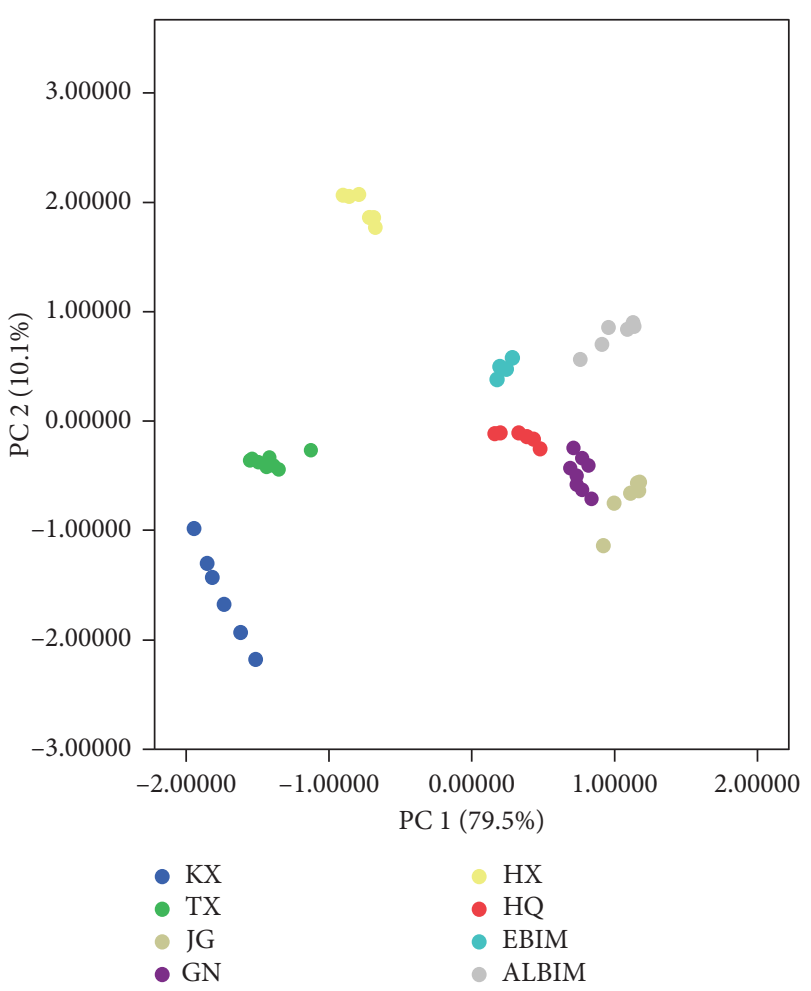

FIGURE 3: Discrimination of PCA plots for different C. songaricum samples.

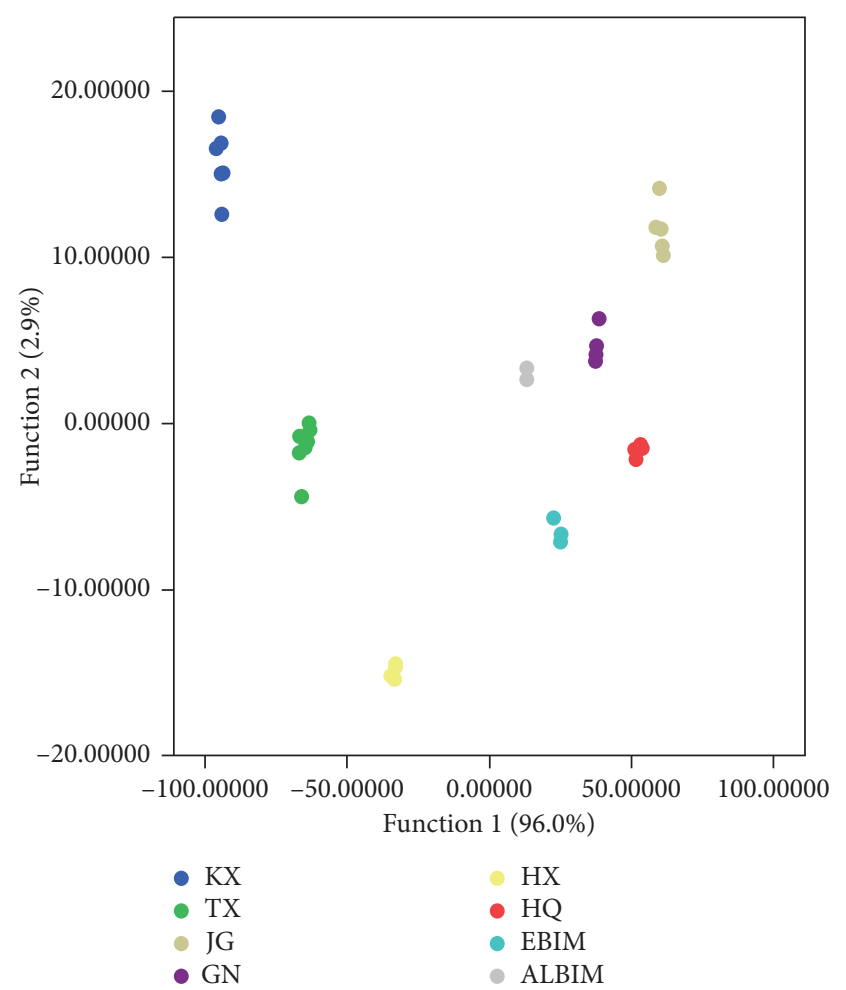

FIGURE 4: Discrimination of LDA plots for different C. songaricum samples.

groups of C. songaricum samples can be classified precisely using the LDA model. On this basis, we used the LDA model to predict the geographical origin of several unknown $C$.
TABLE 2: Confusion matrix for LDA prediction method of $C$. songaricum samples from different producing areas.

\begin{tabular}{lcccccccc}
\hline Actual & \multicolumn{7}{c}{ Predicted } \\
\hline & KX & TX & JG & GN & HX & HQ & EBIM & ALBIM \\
KX & 6 & & & & & & & \\
TX & & 6 & & & & & & \\
JG & & & 6 & & & & & \\
GN & & & & 6 & & & & \\
HX & & & & & 6 & & & \\
HQ & & & & & & 6 & & \\
EBIM & & & & & & 6 & 6 \\
ALBIM & & & & & & & 6 \\
\hline
\end{tabular}

songaricum samples. A prediction model with $100 \%$ degree of accuracy was achieved. We present for the first time a method for the discrimination and geographical origin prediction of C. songaricum from different growing areas in China according to their flavor by an electronic tongue. The operations of data acquisition and processing are simpler and more convenient than the traditional chemical methods. The acquired information can be used for evaluating the quality of $C$. songaricum growing in China according to our previous work. Moreover, the identification and quality analysis method presented by us will be helpful for further study of $C$. songaricum. Further efforts should be focused on investigating the connection between the flavor and chemical constituents of $C$. songaricum samples and correlating electronic tongue signals with human perceptions of taste.

\section{Data Availability}

The data used to support the findings of this study are available from the corresponding author upon request.

\section{Conflicts of Interest}

The authors declare that they have no conflicts of interest regarding the publication of this article.

\section{Acknowledgments}

This study was supported by grants from the National Natural Science Foundation of China (81473315), Public Welfare Scientific Research Project of State Administration of Traditional Chinese Medicine (201507004-2-1), CAMS Innovation Fund for Medical Sciences (no. 2016-I2M-3015), and State Administration of Foreign Experts Affairs P.R. China (no. T2017052).

\section{References}

[1] Z. Cui, Z. Guo, J. Miao et al., "The genus Cynomorium in China: an ethnopharmacological and phytochemical review," Journal of Ethnopharmacology, vol. 147, no. 1, pp. 1-15, 2013.

[2] X. Zhang, C. Gu, B. Ahmad, and L. Huang, "Optimization of extract method for Cynomorium songaricum Rupr. By 
response surface methodology," Journal of Analytical Methods in Chemistry, vol. 2017, Article ID 6153802, 7 pages, 2017.

[3] J. Chen, H. Wong, and K. Ko, "Ursolic acid-enriched herba cynomorii extract induces mitochondrial uncoupling and glutathione redox cycling through mitochondrial reactive oxygen species generation: protection against menadione cytotoxicity in H9c2 cells," Molecules, vol. 19, no. 2, pp. 1576-1591, 2014.

[4] S. Jin, Eerdunbayaer, A. Doi et al., "Polyphenolic constituents of Cynomorium songaricum Rupr. And antibacterial effect of polymeric proanthocyanidin on Methicillin-resistant Staphylococcus aureus," Journal of Agricultural and Food Chemistry, vol. 60, no. 29, pp. 7297-7305, 2012.

[5] S. Tuvaanjav, H. Shuqin, M. Komata et al., "Isolation and antiviral activity of water-soluble Cynomorium songaricum Rupr. Polysaccharides," Journal of Asian Natural Products Research, vol. 18, no. 2, pp. 159-171, 2016.

[6] H. C. Meng, S. Wang, Y. Li, Y. Y. Kuang, and C. M. Ma, "Chemical constituents and pharmacologic actions of Cynomorium plants," Chinese Journal of Natural Medicines, vol. 11, no. 4, pp. 321-329, 2013.

[7] C. Ma, N. Nakamura, H. Miyashiro, M. Hattori, and K. Shimotohno, "Inhibitory effects of constituents from Cynomorium songaricum and related triterpene derivatives on HIV-1 protease," Chemical \& Pharmaceutical Bulletin, vol. 47, no. 2, pp. 141-145, 1999.

[8] C. M. Ma, Y. Wei, Z. G. Wang, and M. Hattori, "Triterpenes from Cynomorium songaricium-analysis of HCV protease inhibitory activity, quantification, and content change under the influence of heating," Journal of Natural Medicines, vol. 63, no. 1, pp. 9-14, 2009.

[9] F. R. Yu, Y. Liu, Y. Z. Cui et al., "Effects of a flavonoid extract from Cynomorium songaricum on the swimming endurance of rats," American Journal of Chinese Medicine, vol. 38, no. 1, pp. 65-73, 2010.

[10] S. Zheng, W. Ren, and L. Huang, "Geoherbalism evaluation of radix Angelica sinensis based on electronic nose," Journal of Pharmaceutical and Biomedical Analysis, vol. 105, pp. 101106, 2015.

[11] M. Goodarzi, P. J. Russell, and Y. V. Heyden, "Similarity analyses of chromatographic herbal fingerprints: a review," Analytica Chimica Acta, vol. 804, pp. 16-28, 2013.

[12] X. Cetó, C. Apetrei, M. del Valle, and M. L. RodríguezMéndez, "Evaluation of red wines antioxidant capacity by means of a voltammetric e-tongue with an optimized sensor array," Electrochimica Acta, vol. 120, pp. 180-186, 2014.

[13] X. Cetó, J. Capdevil, A. Puig-Pujor, and M. del Valle, "Cava wine authentication employing a voltammetric electronic tongue," Electroanalysis, vol. 26, no. 7, pp. 1504-1512, 2014.

[14] X. Cetó, J. M. Gutiérrez, M. Gutiérrez et al., "Determination of total polyphenol index in wines employing a voltammetric electronic tongue," Analytica Chimica Acta, vol. 732, pp. 172-179, 2012.

[15] M. del Valle, "Electronic tongues employing electrochemical sensors," Electroanalysis, vol. 22, pp. 1539-1555, 2010.

[16] L. Escuder-Gilabert and M. Peris, "Review: highlights in recent applications of electronic tongues in food analysis," Analytica Chimica Acta, vol. 665, no. 1, pp. 15-25, 2010.

[17] J. M. Gutiérrez, Z. Haddi, A. Amari et al., "Hybrid electronic tongue based on multisensor data fusion for discrimination of beers," Sensors and Actuators B: Chemical, vol. 177, pp. 989-996, 2013.
[18] F. Winquist, "Voltammetric electronic tongues-basic principles and applications," Microchimica Acta, vol. 163, no. 1-2, pp. 3-10, 2008.

[19] Y. Vlasov, A. Legin, A. Rednitskaya, C. Di Natale, and A. D’Amico, "Nonspecific sensor arrays ("electronic tongue") for chemical analysis of liquids (IUPAC Technical Report)," Pure and Applied Chemistry, vol. 77, no. 11, pp. 1965-1983, 2009.

[20] A. K. Deisingh, D. C. Stone, and M. Thompson, "Applications of electronic noses and tongues in food analysis," International Journal of Food Science and Technology, vol. 39, no. 6, pp. 587-604, 2004.

[21] M. Ringner, "What is principal component analysis," Nature Biotechnology, vol. 26, no. 3, pp. 303-304, 2008.

[22] A. Maćkiewicz and W. Ratajczak, "Principal components analysis (PCA)," Computers \& Geosciences, vol. 19, no. 3, pp. 303-342, 1993.

[23] R. A. Fisher, "The use of multiple measurements in taxonomic problems," Annals of Eugenics, vol. 7, no. 2, pp. 179-188, 1936.

[24] P. N. Belhumeur, J. P. Hespanha, and D. J. Kriegman, "Eigenfaces vs. Fisherfaces: recognition using class specific linear projection," IEEE Transactions on Pattern Analysis and Machine Intelligence, vol. 19, no. 7, pp. 711-720, 1997.

[25] S. Dudoit, J. Fridltand, and T. P. Speed, "Comparison of discrimination methods for the classification of tumors using gene expression data," Journal of the American Statistical Association, vol. 97, no. 457, pp. 77-87, 2002.

[26] D. L. Swets and J. J. Weng, "Using discriminant eigenfeatures for image retrieval," IEEE Transactions on Pattern Analysis and Machine Intelligence, vol. 18, no. 8, pp. 831-836, 1996.

[27] C. A. Blanco, R. de la Fuenta, I. Caballero, and M. L. Roderíguez-Méndez, "Beer discrimination using a portable electronic tongue based on screen-printed electrodes," Journal of Food Engineering, vol. 157, pp. 57-62, 2015. 

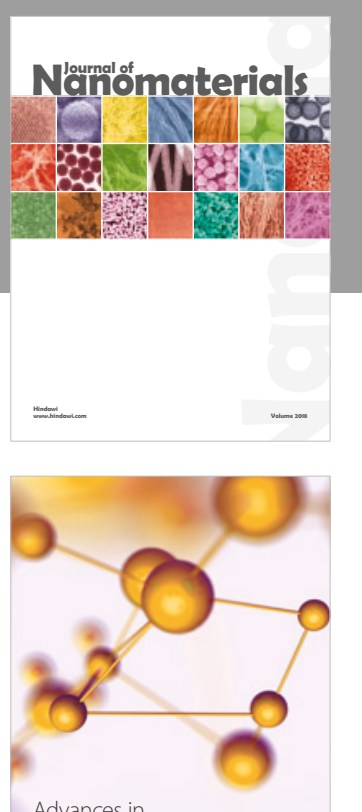

Physical Chemistry
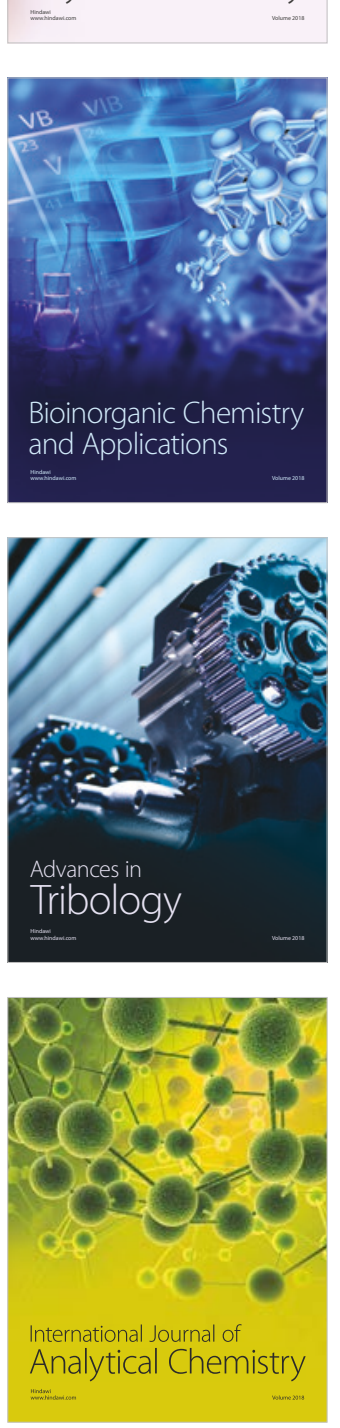

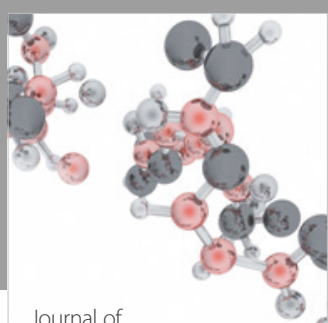

Analytical Methods

in Chemistry

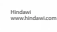

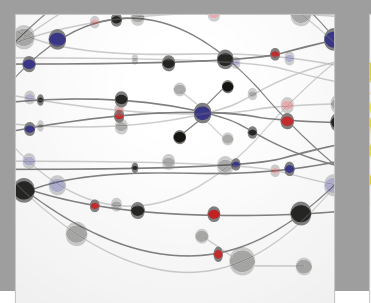

The Scientific World Journal

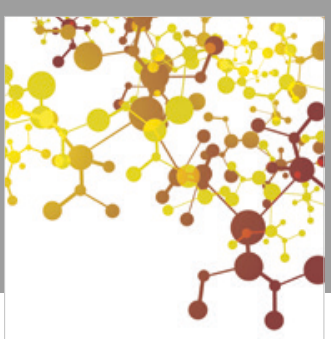

Journal of

Applied Chemistry
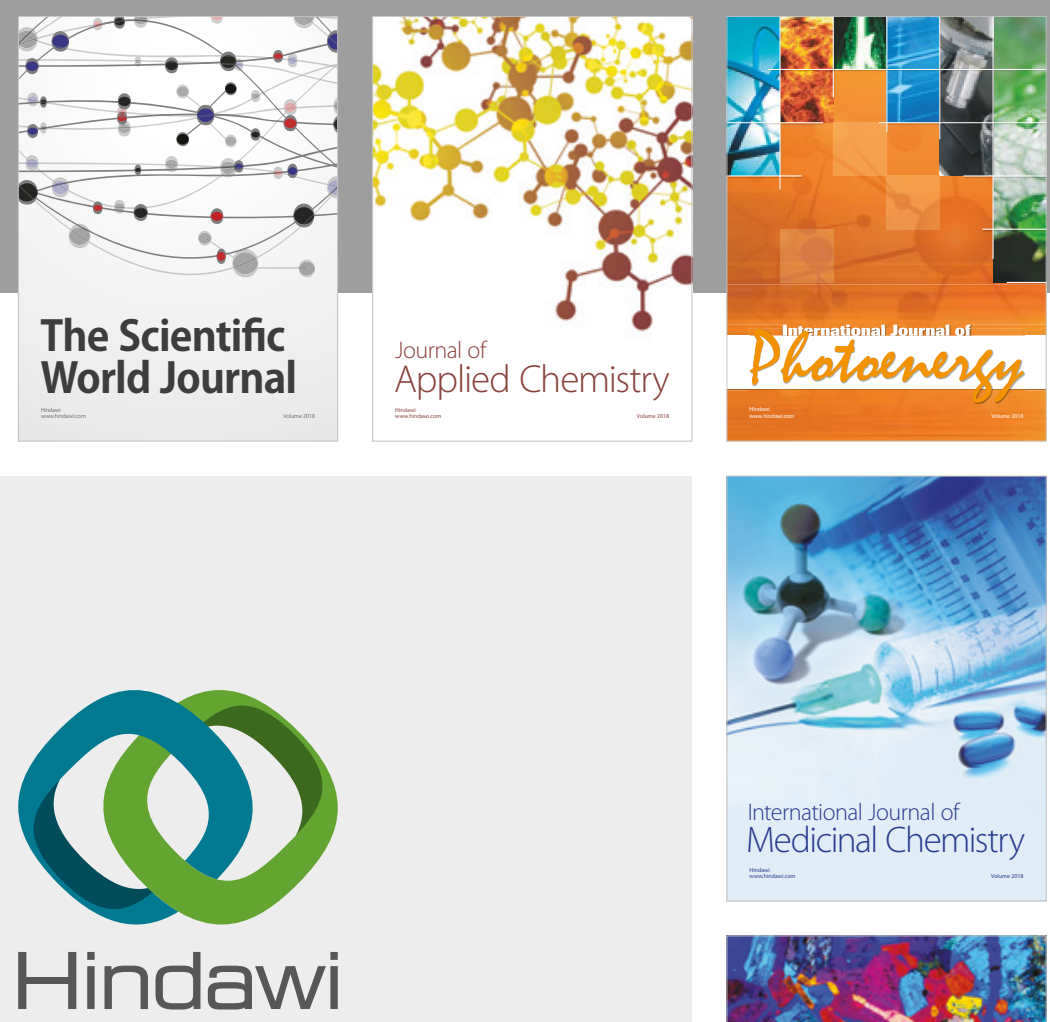

Submit your manuscripts at

www.hindawi.com
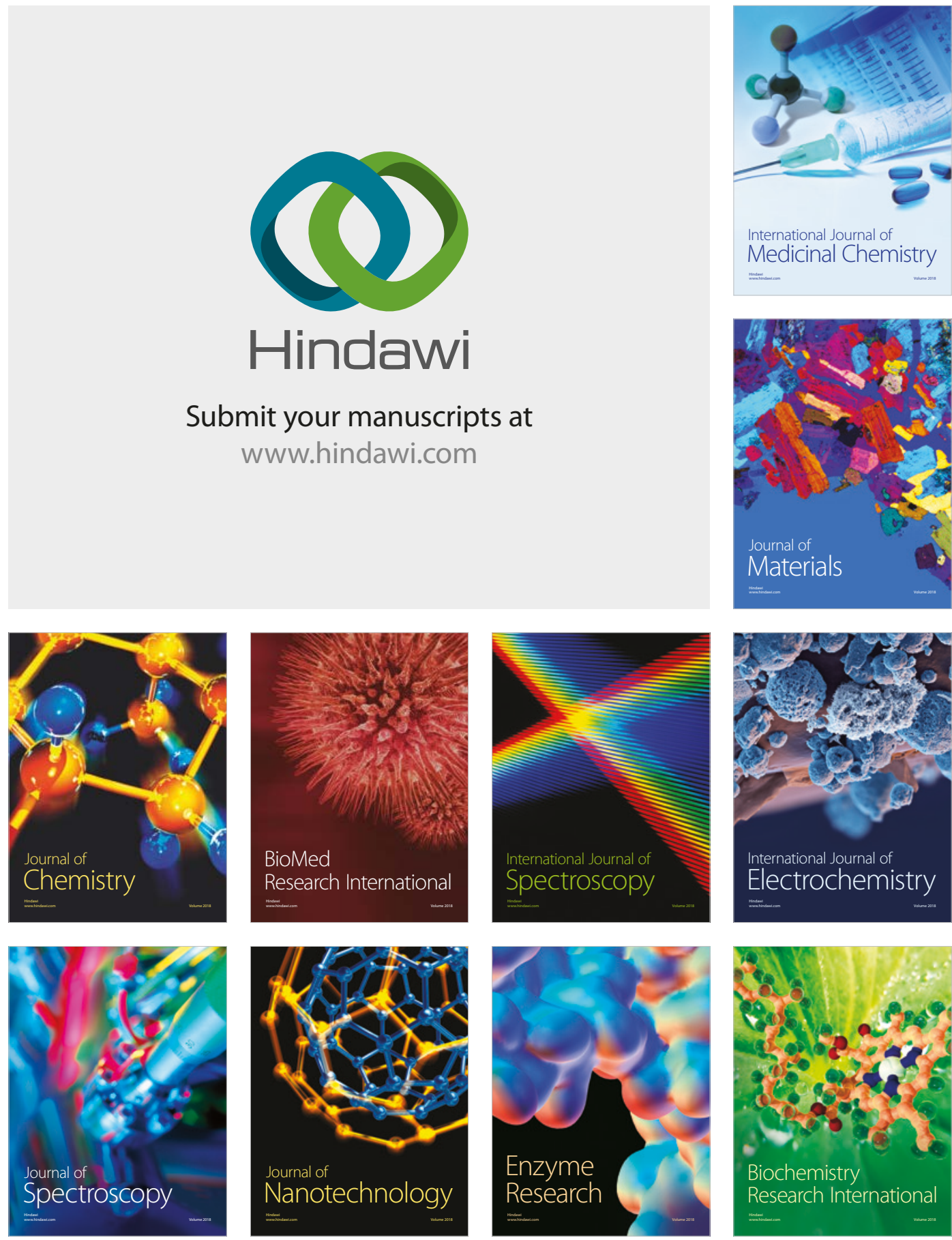
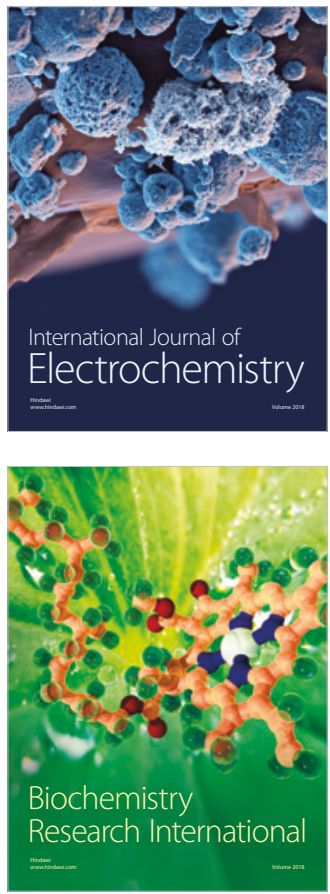